\title{
Solar Corona and Wind
}

\author{
J. F. MCKeNZIE \\ Max-Planck-Institut für Aeronomie, D-3411 Katlenburg-Lindau, F.R.G.
}

(Received August 30, 1990; Revised November 12, 1990)

This paper provides a brief review of basic solar wind theory and makes use of the Laval nozzle analogy to elucidate the characteristic features of a solar wind driven by thermal and Alfvén wave pressure. It is argued that there is a considerable body of evidence in favour of wave acceleration of high speed solar wind. Moreover we advocate that short period waves $(<1$ second) are responsible for heating and accelerating the wind in coronal holes. These waves may be generated by small scale reconnection events (microflares) in regions where the magnetic field is not strictly unipolar.

\section{Introduction}

More than three decades after Parker formulated the first theoretical model of the solar wind the basic physical processes involved in heating the corona and driving the wind are still not properly understood. The original idea that the high temperature of the corona was maintained by dissipation of waves generated in turbulent photospheric layers has, to some extent, given way to other mechanisms. Prominent among these are dissipative heating by currents sheets (PARKER, 1987, 1990) and resonant absorption (KUPERUS et al., 1981; HOLLWEG, 1984; DAVILA, 1987) both of which mechanisms are spontaneously generated by the continuous random motion of the footprints of bipolar magnetic fields which gives rise to twisting and braiding of the flux tubes. Although these mechanisms may encounter some success in explaining high coronal temperatures $\left(\sim 1.5 \times 10^{6} \mathrm{~K}\right)$ and loop brightening, they do not operate effectively in open field regions-such as coronal holes from which high speed solar wind is believed to emanate and therefore other mechanisms must be at work in heating the plasma in coronal holes and driving the high speed solar wind.

Observations (NEUGEBAUER, 1983) indicate that the solar wind exhibits two distinct states, namely high speed streams characterized by remarkably smooth properties of the plasma and its composition on the one hand, and on the other, low speed streams whose properties are highly variable with considerable fluctuations in density and composition. The highly variable characteristics of low speed streams may result from the transient opening and closing of magnetic flux tubes in non-equilibrium regions in the corona. The distinguishing feature of high speed streams is that they are associated with long-term coronal hole regions and, in the streams at least, have one sense of magnetic field representing the excess flux of that sign in the coronal hole itself. Therefore we adopt the viewpoint that a theory of the solar wind must primarily be directed at explaining high speed streams (e.g. Kopp, 1981; AXFORD, 1977, 1980, 1985). 
Energy budget arguments show that high speed solar wind streams $(\sim 700 \mathrm{~km} / \mathrm{sec}$ at 1 A.U.) require effective coronal temperatures in excess of 10 million degrees which is a strong argument in favour of wave acceleration of the wind. There is also a considerable body of other evidence which supports this view, namely: the minor ions move faster and are hotter than the protons; all species tend to have their perpendicular temperatures greater than their parallel temperatures; Alfvén waves observed at 0.3 A.U. propagate away from the sun indicating that the sun itself is a source of such waves. We stress here however that the Alfvén waves usually considered in the solar wind having periods between $10^{2}$ and $10^{5} \mathrm{sec}$ are unlikely to play any role in heating and accelerating the wind and that there are good reasons to believe that much shorter period (from $10^{-2}$ to $1 \mathrm{sec}$ ) waves must be involved.

The usual conservation laws for steady flow along a stream tube in the presence of waves, which are described by a wave energy exchange equation allowing for driving and heating the flow, lead to a "wind equation" which governs the acceleration of the solar wind. For a spherically symmetric flow in which the area of a stream tube varies as $r^{2}$ this equation exhibits a single critical point (of the saddle type) which smoothly connects a subsonic flow inside it to the supersonic flow outside required for equilibrium with the small interstellar pressure at large distances from the sun. It is instructive to make explicit use of the analogy between the solar wind and fluid flow in a Laval nozzle by deriving the equivalent shape of the nozzle. By ensuring that the flow goes sonic at the throat of the nozzle it is possible to deduce how the mass flux "discharged" and the terminal flow speed depend on conditions in the "reservoir" (corona) and the equation of state for the gas (the adiabatic index $\gamma$ ). It turns out that for Alfvén wave pressure the appropriate $\gamma=1 / 2$ which, in the solar wind context, gives rise to the qualitative idea that Alfvén waves drive fast, dilute winds whereas plasma pressure (for which $\gamma \geq 1$ ) drives slow, sluggish, dense winds. However in the more general context of stellar winds it is emphasized that, for the same ratio of escape speed to equivalent sound speed in the corona, Alfvén waves are much more eflfective in driving high speed massive outflows than thermal plasma pressure. It is also interesting to note that an analysis of the changes induced in a steady flow in a Laval nozzle by the addition of heat and momentum can be used to confirm in an elegant way the findings of LEER and HOLZER (1980) concerning how such effects alter the mass flux and terminal speed of the solar wind.

Here we attribute the initial heating and acceleration of the solar wind in a coronal hole to high frequency, short period waves. Although there are no obvious sources of such waves in the chromosphere and photosphere it is possible that they may be generated by small scale reconnection events (microflares) triggered by emerging flux loops in regions where the field is not strictly unipolar. Such waves with periods between $10^{-2}$ and $1 \mathrm{sec}$ would encounter ion cyclotron resonances somewhere between $3 R_{\odot}$ and $30 R_{\odot}$. Although there is as yet no satisfactory theory of this resonance in a multi-component flowing plasma it is fairly certain that cyclotron absorption will result in large perpendicular temperatures and velocities with preferential acceleration and heating of the heavier minor ions.

\section{Basic Solar Wind Theory}

The "wind equation" governing the radial acceleration of the solar wind follows from the conservation laws of mass, momentum and energy. Within a one-fluid framework these laws, for steady plasma flow driven along a radially directed stream tube of cross sectional area $A(r)$ by plasma $\left(p_{\mathrm{g}}\right)$ and wave $\left(p_{\mathrm{w}}\right)$ pressures, may be written 


$$
\begin{aligned}
& \rho u A=\text { const., } \\
& \rho u \frac{\mathrm{d} u}{\mathrm{~d} r}=-\frac{\mathrm{d}}{\mathrm{d} r}\left(p_{\mathrm{g}}+p_{\mathrm{w}}\right)-\rho \frac{G M_{\odot}}{r^{2}}, \\
& \frac{1}{A} \frac{\mathrm{d}}{\mathrm{d} r}\left\{A \rho u\left[\frac{1}{2} u^{2}+\frac{\gamma_{\mathrm{g}}}{\gamma_{\mathrm{g}}-1} \frac{p_{\mathrm{g}}}{\rho}-\frac{G M_{\odot}}{r}\right]+A\left(F_{\mathrm{w}}+F_{\mathrm{c}}\right)\right\}=Q,
\end{aligned}
$$

where $\rho$ and $u$ are respectively the plasma density and radial velocity, $\gamma_{\mathrm{g}}$ is the adiabatic index (=5/3 for ionized hydrogen), $F_{\mathrm{c}}$ is the heat conduction flux ( $\left.\propto T^{5 / 2} \mathrm{~d} T / \mathrm{d} r\right), F_{\mathrm{w}}$, is a wave flux and $M_{\odot}$ is the mass of the Sun. This system is completed by wave energy exchange equations which allow for the work done on the wind by wave pressures and the dissipative heating of the plasma by wave damping as represented by the term $Q$. For example for outward propagating Alfvén waves the exchange equation may be written

$$
\begin{aligned}
& \frac{1}{A} \frac{\mathrm{d}}{\mathrm{d} r}\left(A F_{\mathrm{w}}\right)=u \frac{d p_{\mathrm{w}}}{\mathrm{d} r}-Q, \\
& F_{\mathrm{w}}=p_{\mathrm{w}}(3 u+2 V),
\end{aligned}
$$

in which the Alfvén speed $V$ is given by

$$
V=B / \sqrt{\mu_{o} \rho}
$$

where the flux of the radial magnetic field $B$ is conserved along a stream (flux) tube, i.e.

$$
B A=\text { const }
$$

Eq. (4a) may be cast in the useful form

$$
\frac{1}{A} \frac{\mathrm{d}}{\mathrm{d} r}\left[A \frac{(u+V)^{2}}{V} 2 p_{\mathrm{w}}\right]=-\frac{(u+V)}{V} Q,
$$

which describes the evolution of the wave action in the presence of dissipation. Similarly Eqs. (1)-(4) imply that the plasma entropy evolves according to

$$
\frac{u}{(\gamma-1)}\left(\frac{\mathrm{d} p_{\mathrm{g}}}{\mathrm{d} r}-c_{\mathrm{g}}^{2} \frac{\mathrm{d} \rho}{\mathrm{d} r}\right)=Q-\frac{1}{A} \frac{\mathrm{d}}{\mathrm{d} r}\left(A F_{\mathrm{c}}\right),
$$

where $c_{\mathrm{g}}{ }^{2}$, the square of the isentropic gas sound speed, is given by

$$
c_{\mathrm{g}}^{2}=\gamma_{\mathrm{g}} p_{\mathrm{g}} / \rho
$$


When dissipation is neglected, the two adiabatic integrals arising from Eqs. (5) and (6) namely

$$
\frac{p_{\mathrm{g}}}{\rho^{\gamma \mathrm{g}}}=\text { const. and } A p_{\mathrm{w}} \frac{(u+V)^{2}}{V}=\text { const. }
$$

enable us to derive the following wind equation (BELCHER, 1971; ALAZRAKI and COUTURIER, 1971; BELCHER and OLBERT, 1975)

$$
\begin{aligned}
& \frac{\mathrm{d} u}{\mathrm{~d} r}=\frac{u\left[V_{\mathrm{p}}^{2}\left(\frac{r}{A} \frac{\mathrm{d} A}{\mathrm{~d} r}\right)-\frac{G M_{\odot}}{r}\right]}{r\left(u^{2}-v_{\mathrm{p}}^{2}\right)}, \\
& v_{\mathrm{p}}^{2}=\frac{\partial}{\partial \rho_{\mathrm{ad}}}\left(p_{\mathrm{g}}+p_{\mathrm{w}}\right)=c_{\mathrm{g}}^{2}+c_{\mathrm{A}}^{2}, \\
& c_{\mathrm{A}}^{2} \equiv p_{\mathrm{w}}(3 u+V) / 2 \rho(u+V) .
\end{aligned}
$$

The wind equation exhibits critical points where sonic flow $\left(u=v_{\mathrm{p}}\right)$ is achieved simultaneously with the vanishing of the numerator. For a spherically symmetric variation of the stream tube $\left(A \propto r^{2}\right)$ only one critical point exists and this smoothly connects a subsonic flow inside the critical point to a supersonic flow outside. (In rapidly diverging flow such as may prevail in a coronal hole more complicated topologies, possibly involving standing inner shocks, can arise (e.g. LEER and HOLZER, 1990)).

Characteristic properties of the supersonic solar wind predicted by the wind equation such as the mass flux and terminal speed depend on a number of parameters such as coronal conditions (e.g. the temperature and wave amplitude) and the nature and distribution of the dissipative heating mechanisms. These properties have been elucidated in a number of modelling studies and the general conclusions are: (I) plasma pressure drives low speed. dense winds; (II) Alfvén wave pressure drives high speed, dilute winds; (III) the mass flux, terminal speed and temperature depend sensitively on the location of heating and momentum deposition. These features can be qualitatively understood by using the Laval nozzle analogy.

\section{The Solar Wind as a Laval Nozzle}

It has been noted that the dynamics of the solar wind has a certain analogy to gas flow in a Laval nozzle (PARKER, 1963). In the simplest case of a polytropic description, in which the gas or wave pressure $p \propto \rho^{\gamma}$, it can be readily shown that the solar wind equations are completely equivalent to flow in a Laval nozzle whose cross-sectional area $S(r)$ varies with distance $r$ along its axis as

$$
\frac{S(r)}{S\left(r_{0}\right)}=\frac{A(r)}{A\left(r_{0}\right)}\left[\frac{(\gamma-1)}{c_{0}^{2}}\left(E_{0}+\phi(r)\right)\right]^{\alpha}, \quad \alpha \equiv \frac{(\gamma+1)}{2(\gamma-1)}
$$


where $\phi(r)$ is the gravitational potential, the suffix " 0 " refers to the state at some reference level (the reservoir) - in our case the corona so that $E_{0}$ is the energy per unit mass and $c_{0}$ is the sound speed there. The topology of the integral curves of the Mach number $M(=u / c)$ versus distance $r$ follows directly from the equations of motions and is described by

$$
\frac{\left(1+(\gamma-1) M^{2} / 2\right)^{\alpha}}{M}=\frac{1}{M_{0}} \frac{S(r)}{S\left(r_{0}\right)} \text {. }
$$

For the case of spherically symmetric flow $\left(A \propto r^{2}\right)$ in the presence of a gravitating point mass $\left(\phi=G M_{\odot} / r\right)$ the equivalent Laval nozzle exhibits a single throat (a minimum in $S(r)$ ) at $r_{\mathrm{c}}$ where

$$
\frac{r_{\mathrm{c}}}{r_{\mathrm{o}}}=\frac{(5-3 \gamma) X / 8}{\left[1-(\gamma-1)\left(X-M_{\mathrm{o}}^{2}\right) / 2\right]}, \quad X \equiv v_{\mathrm{e}}^{2} / c_{0}^{2}, \quad v_{\mathrm{e}}=\sqrt{2 G M_{\odot} / r}
$$

The critical outflow solution is obtained by ensuring that the flow is sonic $(M=1)$ at the throat $\left(r=r_{\mathrm{c}}\right)$ and this requirement in Eq. (9) yields the associated outflow mass flux / unit area $J_{\mathrm{o}}=\rho_{\mathrm{o}} c_{\mathrm{o}} M_{\mathrm{o}}$ in which $M_{\mathrm{o}}$ is the subsonic solution of the equation

$$
\left(M_{\mathrm{o}} /(X / 4)^{2}\right)^{2 n}-n M_{\mathrm{o}}^{2}=1+3 n-n X, \quad n \equiv(\gamma-1) /(5-3 \gamma)
$$

The location of the throat and the mass flux discharged depend sensitively on the "coronal" parameters $\gamma$ (the equation of state) and $X$ (a measure of the ratio of the binding gravitational energy to the internal energy). The behaviour of both the subsonic and supersonic solutions of this equation, which includes accretion as well as stellar wind flows, is shown in Fig. 1 as

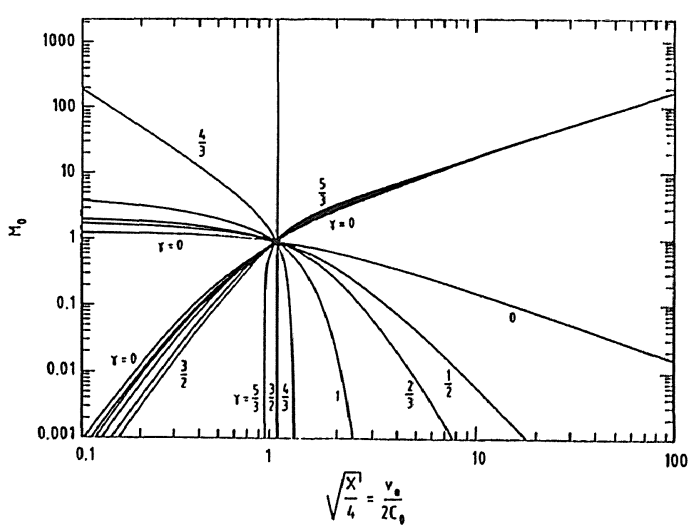

Fig. 1. Solutions of the mass flux Eq. (11) as plots of the coronal Mach number $M_{\mathrm{o}}$ versus $v_{\mathrm{e}} / 2 c_{\mathrm{o}}$ for various values of the adiabatic index $\gamma$. The mass flux/unit area is $\rho_{0} c_{0} M_{0}$ where, for given coronal conditions, $M_{0}$ lies on the subsonic $\left(M_{0}<1\right)$ branch of the curves. 
$M_{\mathrm{o}}$ versus $\sqrt{X / 4}$ for various values of $\gamma$.

If $\gamma=5 / 3$ (as is the case for ionized hydrogen) the throat occurs at the origin and a smooth subsonic - supersonic flow can only be achieved by including extended heating of the corona, for example either by joining $\gamma=1$ solutions for the inner flow to $\gamma=5 / 3$ outer solutions (PARKER, 1963), or by solving the equations with $\gamma=5 / 3$ and a suitable distribution of $Q$ (HOLZER and AXFORD, 1970). For the special case $\gamma=1$, corresponding to an isothermal expansion, the critical point occurs at $r_{\mathrm{c}} / r_{\mathrm{o}}=X / 4$ which corresponds to $4 R_{\odot}$ for a $1.5 \times 10^{6} \mathrm{~K}$ corona. In this case the $\gamma \rightarrow 1$ limit of Eq. (11) yields

$$
J_{0} \simeq \rho_{0} c_{0}(X / 4)^{2} \exp (3 / 2-X / 2)
$$

which exhibits exponential sensitivity of $J_{0}$ to the coronal temperature $T_{\mathrm{o}}$ through $c_{\mathrm{o}}{ }^{2}=$ $2 k T_{\mathrm{o}} / m_{\mathrm{p}}$ (LEER et al., 1982; WITHBROE, 1988). Moreover the wind speed at 1 A.U. turns out to be somewhat low of the order of $400 \mathrm{~km} / \mathrm{sec}$ in an isothermal model with coronal temperatures in the range 1 to $1.5 \times 10^{6} \mathrm{~K}$. Solutions with $1<\gamma<5 / 3$ display a similar behaviour of the terminal speed and the mass flux, provided of course $X<2 /(\gamma-1)$ to ensure an outflow along with the additional requirement $\gamma \leq 3 / 2$ to yield an accelerating flow.

On the other hand the flow characteristics for the "unusual" case, $\gamma<1$, reveal a different behaviour. The equivalent nozzle area variation is more rapid with a wider throat which occurs closer to the solar surface at

$$
\frac{r_{\mathrm{c}}}{r_{\mathrm{o}}} \simeq \frac{(5-3 \gamma)}{4(1-\gamma)}, \quad \frac{X}{4} \gtrsim 1
$$

The associated mass flux $J_{0}$, obtained from the approximate subsonic root of (11) depends on $X$ and $\gamma$ in the following way,

$$
J_{\mathrm{o}} \simeq\left(\frac{1}{4 m}\right)^{1 / 2 m} \frac{\rho_{\mathrm{o}} c_{\mathrm{o}}}{(X)^{1 / 2 m-2}}, \quad m \equiv \frac{(1-\gamma)}{(5-3 \gamma)}
$$

Although "unusual" it is relevant to consider the case $\gamma<1$. The conservation of wave action integral (7) shows that in sub-Alfvénic flow $\left(u\left\langle\langle V\rangle p_{\mathrm{w}} \propto \rho^{1 / 2}\right.\right.$ whereas in super-Alfvénic flow $p_{w} \propto \rho^{3 / 2}$-thus Alfvén waves behave roughly like a fluid whose adiabatic index varies from $1 / 2$ to $3 / 2$. A similar result prevails in cosmic ray driven winds where the adiabatic index for the cosmic ray pressure equation of state varies between 2/3 and 4/3 (MCKENZIE et al., 1987; BREITSCHWERDT et al., 1990). Therefore for stellar winds driven by Alfvén waves where the sonic point is achieved while the flow remains sub-Alfvénic it is a good approximation to use an equivalent $\gamma$ of $1 / 2$ in Eqs. (13) and (14) which then yield,

$$
\frac{r_{\mathrm{c}}}{r_{\mathrm{o}}} \simeq \frac{1}{4}, \quad J_{\mathrm{o}}=\left(\frac{7}{4}\right)^{7 / 2} \rho_{\mathrm{o}} \frac{c_{\mathrm{o}}^{4}}{v_{\mathrm{e}}^{3}}, \quad c_{\mathrm{o}}^{2}=\frac{1}{2} \frac{p_{\mathrm{wo}}}{\rho_{\mathrm{o}}}=\frac{1}{4} u_{\mathrm{wo}}^{2},
$$

where $u_{\mathrm{wo}}$ is the amplitude of the Alfvén waves at base of the corona. It follows from energy flux conservation that the terminal speed $u_{\infty}$ of such a wind is given by 


$$
\frac{u_{\infty}}{v_{\mathrm{e}}} \simeq 2 \sqrt{2}\left(\frac{4}{7}\right)^{7 / 2} \frac{\sqrt{V_{\mathrm{o}} \nu_{\mathrm{e}}}}{c_{\mathrm{o}}} .
$$

These asymptotic relations show that moderate wave amplitudes or wave pressures can drive very rapidly moving winds with ultimate speeds in excess of the escape speed but with an accompanying mass flux output which is rather low. This behaviour contrasts with a $\gamma \geq 1$ solar wind which tends to be dense and sluggish.

\section{Solar Wind Models and Parameter Studies}

Parker's original solar wind model has been considerably modified in attempts to realistically model the characteristic features of the solar wind. This is usually done by choosing realistic parameters at the coronal base and evaluating the effects of energy (and even momentum) addition on the flow. In particular we note LEER and HOLZER (1980) who found that energy addition in the subsonic region increases the solar wind mass flux but either has a negligible effect (for heat addition) or significantly reduces (for momentum addition) the solar wind speed at 1 A.U. Conversely in the supersonic region energy addition can significantly increase the flow speed at 1 A.U. Similarly it is found that momentum addition in the subsonic (supersonic) region leads to a decrease (increase) in the asymptotic flow speed, and an increase in the mass flux when added in the subsonic portion of the flow. It is obvious that the mass flux cannot be affected by processes taking place beyond the sonic point of the flow.

These findings can be understood within the context of the changes induced in a steady flow along a tube (or Laval nozzle) by the addition of amounts of heat, $Q$, and momentum, $\mathcal{M}$, over a short segment of the tube. The usual Rankine-Hugoniot relations connecting the flow across unit area of such a segment are

$$
\begin{aligned}
& p-p_{\mathrm{o}}=-J^{2}\left(\tau-\tau_{\mathrm{o}}\right)+\mathscr{M}, \\
& p\left(\tau-\mu^{-2} \tau_{\mathrm{o}}\right)-p_{\mathrm{o}}\left(\tau_{\mathrm{o}}-\mu^{-2} \tau\right)=\mu^{-2}\left(2 Q-\mathscr{M}\left(\tau+\tau_{\mathrm{o}}\right)\right), \\
& J \equiv \rho_{\mathrm{o}} u_{\mathrm{o}}=\rho u, \\
& \mu^{-2} \equiv(\gamma-1) /(\gamma+1),
\end{aligned}
$$

in which the state ahead is denoted by the suffix o. Equation (17b) is the Hugoniot including a "heat of reaction" term on the right hand side and therefore the state behind (with no suffix) the heated segment is given by the intersection between the locus of the Hugoniot curve in the $(p, \tau)$ plane and the momentum line (19a) (see e.g. COURANT and FRIEDRICHS, 1976). Solutions corresponding to strong discontinuities (shocks and strong deflagrations) can be excluded by linearizing the Hugoniot about the upstream state for small amounts of heat, $\Delta Q$, and momentum, $\Delta \mathcal{M}$, which then simplifies to

$$
p-p_{\mathrm{o}}=-J_{\mathrm{c}}^{2}\left(\tau-\tau_{\mathrm{o}}\right)+(\gamma-1)\left(\Delta Q / \tau_{\mathrm{o}}-\Delta \mathcal{M}\right)
$$


in which

$$
J_{\mathrm{c}}=\rho_{\mathrm{o}} c_{0}
$$

is the maximum attainable mass flux/unit area which occurs at the upstream sound speed $c_{0}$. Thus the changes of $\Delta \mathrm{p},\left(p-p_{\mathrm{o}}\right), \Delta \tau,\left(\tau-\tau_{\mathrm{o}}\right), \Delta T,\left(T-T_{\mathrm{o}}\right)$ induced in the flow by the supply of small amounts of heat and momentum are given by

$$
\begin{aligned}
& \Delta p=\left[(\gamma-1) J^{2} \Delta Q / \tau_{\mathrm{o}}-\left((\gamma-1) J^{2}+J_{\mathrm{c}}^{2}\right) \Delta \mathscr{M}\right] /\left(J^{2} / J_{\mathrm{c}}^{2}\right), \\
& \Delta \tau \equiv \Delta u / J\left[-(\gamma-1) \Delta Q / \tau_{\mathrm{o}}+\gamma \Delta \mathcal{M}\right] /\left(J^{2} / J_{\mathrm{c}}^{2}\right), \\
& \Delta T=(\gamma-1)\left[\left(\gamma J^{2} / J_{\mathrm{c}}^{2}-1\right) \Delta Q / \tau_{\mathrm{o}}^{2}-\gamma\left(J^{2} / J_{\mathrm{c}}^{2}\right) \Delta \mathcal{M} / \tau_{\mathrm{o}}\right] /\left(J^{2} / J_{\mathrm{c}}^{2}\right) .
\end{aligned}
$$

Note the flow is subsonic $\left(u_{0}<c_{0}\right)$ or supersonic $\left(u_{\mathrm{o}}>c_{0}\right)$ according as $J<J_{\mathrm{c}}$ or $J>J_{\mathrm{c}}$. Figure 2 provides a graphical interpretation of the first two results (for $\Delta p$ and $\Delta \tau$ ). Thus we see that in subsonic flow the supply of heat $(\Delta Q>0)$ accelerates the flow $(\Delta u$ and $\Delta \tau>0)$ and decreases the pressure $(\Delta p<0)$ whereas in supersonic flow it decelerates the flow $(\Delta u<$ $0)$ and increases the pressure $(\Delta p>0)$. On the other hand momentum addition $(\Delta \mathcal{M}>0)$ in subsonic sections decelerates the flow $(\Delta u<0)$ and increases the pressure $(\Delta p>0)$ while in supersonic flow the converse is true. The expression (18c) for the temperature change shows that for supersonic flow the temperature is increased (decreased) by the supply of heat (momentum); while in subsonic flow $\Delta T$ may be either positive or negative (LANDAU and LIFSHITZ, 1966). Although certain aspects of these results appear counter intuitive they exhibit the well known characteristic features of "weak detonations and deflagrations" in the presence of a heat of reaction. Moreover when these results are applied to steady flow in the equivalent solar wind Laval nozzle to join two sections of adiabatic flow separated by a short segment where energy and momentum is added the Leer and Holzer findings can be confirmed and understood in an elegant way.

It is intriguing that precisely the same analysis goes through when plasma pressure is replaced by Alfvén wave pressure, in which case we have $\gamma \simeq 1 / 2$ in sub-Alfvénic flow, and then, because $\gamma<1$, our conclusions, regarding the changes induced in the flow by the

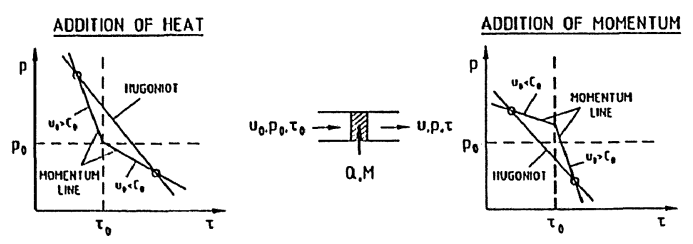

Fig. 2. The intersections between the Hugoniot and the momentum line give the state behind a segment at which small amounts of heat and momentum are added. For example if heat is added to a supersonic flow the pressure is increased and the velocity decreased. 
addition of small amounts of heat and momentum, are reversed with concomitant changes in the flow characteristics at 1 A.U. However to some extent this point is implicitly included in detailed investigations of the acceleration of the solar wind which combine both thermal plasma and Alfvén wave effects, such as in the series of comprehensive studies (see e.g. LEER et al., 1982; ESSER et al., 1986, 1987; ROBERTS, 1989) carried out within the single, twofluid and multi-fluid frameworks where in the latter the ions are regarded as truly minor. Such parameter studies are particularly useful in demonstrating the effects of Alfvén waves on the solar wind density, velocity, proton and minor ion temperature profiles for various coronal conditions and flow geometries. Moreover such studies serve to underline the importance of observations of the electron density, Lyman alpha line, and coronal minor ion spectral lines in placing constraints on the wave amplitudes and the processes involved in the acceleration of the solar wind.

It should be emphasized that the processes involved in heating the corona and the phenomenon of the solar wind are fundamentally related (see e.g. AXFORD, 1985) and the different regions namely the transition region, the corona and the wind should be placed on an equal footing in a self-consistent description. Unified treatments along these lines have been attempted by HOLLWEG (1986) and HOLLWEG and JOHNSON (1988) in which Alfvén waves not only contribute to driving the wind but also dissipatively heat the corona by a turbulent cascade (TU, 1987) at the Kolmogorov rate. These models reproduce the correct qualitative features: a steep rise of the temperature in the transition region; a maximum temperature in the corona in excess of $10^{6} \mathrm{~K}$ and a particle flux at 1 A.U. of around $3.5 \times 10^{8} \mathrm{~cm}^{-2} \mathrm{~s}^{-1}$. However high flow speeds appear to be attainable only if the coronal base pressure is rather low and the random (thermal plus wave) proton velocities are larger than those deduced from Lyman $\alpha$ observations in the inner region between $1.5 R_{\odot}$ and $4 R_{\odot}$.

It is interesting that in solar wind models involving dissipative heating by "short wavelength" Alfvén waves, "long wavelength" compressional instabilities can arise (DOUGHERTY and MCKENZIE, 1990; NAIDU and McKENZIE, 1991), and these may play a role in the large scale heating and dynamics of the solar wind in the supersonic region.

\section{Solar Wind Energy Budget and Constraints}

Observations, summarized in Table 1, indicate that the solar wind exhibits two distinct

Table 1. Comparison of low and high speed streams at 1 A.U.

\begin{tabular}{lll}
\hline & \multicolumn{1}{c}{ Low speed } & \multicolumn{1}{c}{ High speed } \\
\hline Speed & $<400 \mathrm{~km} / \mathrm{sec}$ & $750 \mathrm{~km} / \mathrm{sec}$ \\
Number density & $10-20 \mathrm{~cm}^{-3}$ & $4 \mathrm{~cm}^{-3}$ \\
Proton temperature & $2 \times 10^{4}-10^{5} \mathrm{~K}$ & $2 \times 10^{5} \mathrm{~K}\left(T_{\perp}>T_{\|}\right)$ \\
Electron temperature & $2 \times 10^{5} \mathrm{~K}$ & $10^{5} \mathrm{~K}$ \\
Energy flux & $2-3 \mathrm{ergs} / \mathrm{cm}^{2} \mathrm{sec}$ & $2-3 \mathrm{ergs} / \mathrm{cm}^{2} \mathrm{sec}$ \\
{$\left[\mathrm{He}^{++} / \mathrm{H}^{+}\right]$} & $10^{-3}-10^{-1}$ & 0.05 \\
& (highly variable) & (uniform) \\
Origin & Transiently open coronal & Long-term open \\
& magnetic field regions and & magnetic field regions \\
& "bubbles" & (coronal holes) \\
\hline
\end{tabular}


states, namely high speed streams characterized by remarkably smooth properties of the plasma and its composition on the one hand, and on the other, low speed streams whose properties are highly variable with considerable fluctuations in density and composition. The highly variable characteristics of low speed streams may result from the transient opening and closing of magnetic flux tubes in the corona and represent non-equilibrium regions. The distinguishing feature of high speed streams is that they are associated with long-term coronal hole regions and, in the streams at least, have one sense of magnetic field representing the excess flux of that sign in the corona itself. It is therefore reasonable to adopt the viewpoint that a wave and plasma pressure driven theory of the solar wind must primarily be directed at explaining high speed streams.

In a steady model of the solar wind, including dissipative processes such as heat conduction, the conservation of the total energy of each proton-electron pair is conveniently expressed in the Bernoulli form

$$
\frac{1}{2} u^{2}+\frac{5}{2} \frac{k}{m}\left(T_{\mathrm{p}}+T_{\mathrm{e}}\right)-\frac{G M_{\odot}}{r}+\frac{F_{\mathrm{w}}}{\rho u}+\frac{F_{\mathrm{c}}}{\rho u}=E_{\mathrm{o}}+q
$$

where $q$ represents the wave dissipation term,

$$
q=\frac{1}{\rho u A} \int_{r_{0}}^{r} A Q \mathrm{~d} r
$$

and $E_{\mathrm{o}}$ is the energy/unit mass at the coronal base. If, for the moment, we neglect $F_{\mathrm{w}}, F_{\mathrm{c}}$ and $q$, we find, for a coronal temperature of $1.5 \times 10^{6} \mathrm{~K}$, that $E_{\mathrm{o}} \sim 1700 \mathrm{eV}$, (gravitational + enthalpy) while at 1 A.U., where the energy is predominantly in streaming, the energy/p-e pair is around $3000 \mathrm{eV}$ (for high speed streams) or $900 \mathrm{eV}$ (for low speed). Thus the total energy required is of the order of $5000 \mathrm{eV}$ for high speed streams $(2600 \mathrm{eV}$ for low speed) which is equivalent to an effective coronal temperature of $11 \times 10^{6} \mathrm{~K}$ (or $6 \times 10^{6} \mathrm{~K}$ for low speed). This is a powerful argument in favour of the need for waves because, except for heat conduction, energy transferred to the corona from below must be associated with mass motions in one form or other. As far as observations are concerned it should be noted that "random" mass motions associated with waves should be apparent through an effective temperature $\left(T_{\text {eff }}\right) \gg 1.5 \times 10^{6} \mathrm{~K}$ where we can define (ESSER et al., 1986)

$$
T_{\text {eff }}=T_{\mathrm{o}}+\frac{2}{3} \frac{m}{2 k}\left\langle u_{\mathrm{w}}^{2}\right\rangle
$$

where $u_{\mathrm{w}}$, is the (averaged) velocity amplitude of the waves. These random (wave) motions should be most apparent in the spectrum of heavier species which have smaller thermal speeds at a given temperature. Again following Esser et al. we can define a non-thermal broadening upper limit for $u_{\mathrm{w}}$ given by

$$
u_{\mathrm{w}} \leq\left(\frac{3}{2}\right)^{\frac{1}{2}} c \frac{\Delta \lambda}{\lambda}\left(1-\left(v_{\mathrm{t}} / v_{\mathrm{e}}\right)^{2}\right)^{1 / 2}
$$


where the broadening of a spectral line centred at wavelength $\lambda$ emitted from an element of mass $m$ and effective temperature $T_{\text {eff }}$ is given by

$$
\frac{\Delta \lambda}{\lambda}=\frac{1}{c}\left(\frac{2 k T_{\mathrm{eff}}}{m}\right)^{1 / 2} .
$$

Using the observation $\Delta \lambda / \lambda=10^{-4}$ for Fe XII at $1242 \AA$, along with the constraint that the coronal temperature should not exceed $\sim 1.5 \times 10^{6} \mathrm{~K}$ (determined from freezing in of charge states) we find that $u_{\mathrm{w}}$ must be less than around $27 \mathrm{~km} / \mathrm{sec}$. Since sound waves have a relatively low group velocity $(<150 \mathrm{~km} / \mathrm{sec})$ and are also quickly damped, thereby contributing to heating the lower corona, they are unlikely to meet the energy requirements of high speed streams. On the other hand Alfvén waves with group velocities of the order of $1400 \mathrm{~km} / \mathrm{sec}$ and velocity amplitudes of $25 \mathrm{~km} / \mathrm{sec}$ meet these requirements and at the same time have only relatively small amplitudes at the base of the corona $\left(B_{\mathrm{w}} / B=u_{\mathrm{w}} / V \simeq .02\right)$ with the result that they will propagate to beyond $10 R_{\odot}$ before suffering any form of nonlinear damping which will tend to saturate $B_{\mathrm{w}} / B$. This has the interesting consequence that even if the plasma temperature decreases we may, provided the number density decreases sufficiently rapidly, expect $T_{\text {eff }}$ to increase beyond a certain height, because $u_{\mathrm{w}} \propto \rho^{-1 / 4}$ by virtue of the $\gamma=1 / 2$ "equation of state" for the wave pressure implied by approximate conservation of the wave energy flux in sub-Alfvénic flow.

Thus the evidence for wave acceleration of the solar wind is strong and may by summarized as follows:

(a) energy arguments leave us with little choice;

(b) purely plasma pressure and heat conduction driven winds have the wrong characteristics-they cannot produce high speed streams;

(c) the minor ions move faster and are hotter than the protons;

(d) all species tend to have their perpendicular temperatures greater than their parallel temperatures which cannot be achieved without preferentially heating perpendicular to the magnetic field as would be the case with cyclotron resonance and damping;

(e) the Alfven waves observed at 0.3 A.U. propagate away from the Sun indicating that the Sun is a source of such waves.

However it should be stressed that the Alfven waves usually considered in the solar wind having periods between $10^{2}$ and $10^{5}$ secs with corresponding wavelengths in the lower corona of $10^{10}$ to $10^{13} \mathrm{~cm}$ are unlikely to play any role in heating and accelerating the solar wind since the local requirements for averaging are not satisfied at these long-wavelengths. In fact, as we argue subsequently, there are good reasons to believe that the important periods are in the range $10^{-3}$ to $10 \mathrm{sec}$ with corresponding wavelengths of 1 to $10^{4} \mathrm{~km}$.

\section{Heating in Coronal Holes}

A recent review by PARKER (1990) on the structure of solar and stellar corona points out that wave dissipation can only play a very minor role in heating the solar corona and concludes instead that the X-ray corona is heated by highly dissipative current sheets which are spontaneously formed by the continuous random motions of the footprints of the magnetic field embedded in the photospheric convection. It should be stressed that this mechanism for coronal heating and others such a resonant absorption (KUPERUS et al., 1981; 
HollWEG, 1984; DAVILA, 1987) are only applicable to bipolar (or closed loop) regions in which the ends of the magnetic field lines are anchored in the solar surface. In open field regions-such as coronal holes-motions of the feet cause twists which simply propagate out along the open field lines at the Alfvén speed with periods matching the period of the motions in the photosphere. As already indicated these long wavelength waves are unlikely to be of any significance in the heating of the corona and the acceleration of the solar wind, and that instead we must look to much shorter period waves, in the range $10^{-3} \mathrm{sec}$ to $10 \mathrm{sec}$, to do the job.

There are no obvious sources of waves having the necessary short wavelengths in the solar chromosphere which may be compared with our own atmosphere in terms of its capacity to make waves. The source of the energy is undoubtedly the photosphere and chromosphere, but the waves required may be produced by secondary processes, such as in reconnection-events (microflares) where the time scale is locally determined. However to have reconnection as a possibility in open field regions it is necessary that the magnetic field not be strictly unipolar; instead it would be sufficient merely to have a preponderance of field with one sign with sufficiently small scales that only the average is measured. In this case one would expect neutral atmospheric motions and turbulence to continually make the magnetic field topology more complex and the corresponding growth of the energy stored in the field to be checked by sporadic releases in the form of microflares. The latter would produce both heating and wave motions with typical periods of the order of the Alfvén wave crossing time of the regions concerned.

The overall chromospheric network pattern (GABRIEL, 1976) which is driven by convective overturning of the photosphere leads naturally to strong concentrations of the magnetic field near the supergranulation cell boundaries. For example a $10 \mathrm{G}$ field in the corona would map to $100 \mathrm{G}$ or more, at the cell boundaries where the length scales are of the order of $10^{3} \mathrm{~km}$ or less. Thus Alfvén wave crossing times of one second or less are readily achievable. An emerging flux loop in such a locally unipolar field would undergo reconnection. Such reconnection events, due to mixed polarity in the predominantly unipolar region gives rise to local heating, proportional to the magnetic field strength, accompanied by upward moving high frequency $(\leq 1 \mathrm{sec})$ waves and downward moving plasma resulting from the contraction of closed loops formed by reconnection at higher altitudes. The latter effect may be the explanation for the prevalence of downward motions observed in the bulk of the emissions from the lower corona and transition region. These motions are resisted by the pressure of the plasma trapped in the reconnected loops and the density of the plasma, as well as the emissivity, increase as the field pulls it into the upper chromosphere. Thus we can expect this effect to occur in coronal holes as well as in closed field regions.

The upward moving waves released in these reconnection events can have periods in the range $10^{-2}$ to $1 \mathrm{sec}$. and therefore it is inevitable that they will undergo gyroresonance with protons and minor ions within a few tens of solar radii from the surface. Figure 3 shows the variation of the cyclotron period as a function of the field strength $B$ from $10 \mathrm{G}$ down to $10^{-2}$ $\mathrm{G}$. Since the wave periods generated by the small scale reconnection events are in the range $10^{-2}$ to $1 \mathrm{sec}$. (i.e. lying above the horizontal broken line) we see that all periods will encounter a gyroresonance, first with the heavier species and then with protons, somewhere between $3 R_{\odot}$ and $30 R_{\odot}$ depending on the nature (monopole or dipole) of the fall off in the field with radius. Cyclotron absorption at these gyroresonances should give rise to large perpendicular ( $\perp$ to $\boldsymbol{B}$ ) velocities and temperatures, accompanied by preferential heating and acceleration of minor ions (MCKENZIE et al., 1979; MCKENZIE and MARSCH, 1982; 


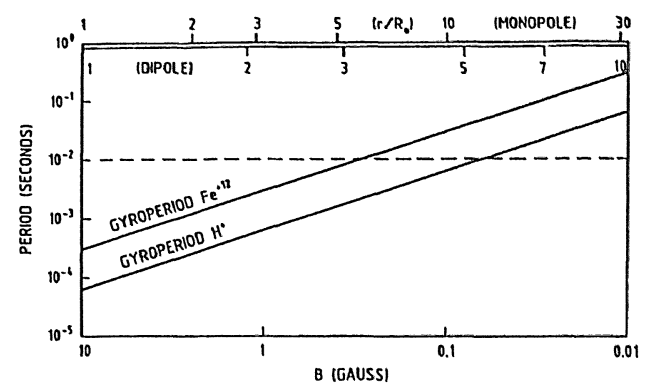

Fig. 3. The gyroperiods of $\mathrm{H}^{+}$and $\mathrm{Fe}^{+12}$ as functions of the magnetic field strength (bottom axis) or solar radius (top axis) for dipole and monopole fall-offs. Short period waves generated by small scale reconnection events, lying above the horizontal broken line will meet gyroresonances with all species somewhere between $3 R_{\odot}$ and $30 R_{\odot}$.

ISENBERG and HOLLWEG, 1983). However it is noted that a fully self-consistent nonlinear theory of gyroresonance in a multi-component flowing plasma is not yet in place.

Nevertheless the scintillation and other observations (KOJIMA et al., 1987; BRUECKNER and BARTOE, 1983; BRUECKNER, 1990; MANDRINI et al., 1990; DERE et al., 1989; DERE, 1990; MARISKA, 1989) lend some support to this overall picture with very strong turbulence being detected in the region close to the sun (say $1.5 R_{\odot}$ to $20 R_{\odot}$ ). The turbulent velocities are of the order of hundreds of $\mathrm{km} / \mathrm{sec}$ at around ten solar radii. The time scales and length scales appear to be consistent with the short period waves we advocate-but this requires careful appraisal. There are also strong indications of the presence of density fluctuations perpendicular to the magnetic field which may or may not be a consequence of magnetoacoustic or higher frequency plasma waves.

It is also necessary to find a process which is capable of heating the coronal electrons to $10^{6} \mathrm{~K}$. It would be convenient and elegant if this were somehow associated with the more important ion heating and hence the parallel electric fields accompanying the ion cyclotron resonances would offer an explanation which at the same time would be linked with the production of the density fluctuations implied by the scintillations. However it is more likely that electron heating is associated with lower hybrid waves-but again this needs to be looked at very carefully.

I have benefitted from discussions with Professors W. I. Axford and C.-Y. Tu, Drs. W.-H. Ip and E. Marsch and I am particularly grateful to Dr. M. K. Dougherty for producing Fig. 1.

\section{REFERENCES}

Alazraki, G. and P. Couturier, Astron. Astrophys., 13, 380, 1971.

AXFoRD, W. I., Study of Travelling Interplanetary Phenomena/1977, 145 pp., D. Reidel, Dordrecht-Holland, 1977.

AXFORD, W. I., Highlights of Astronomy, 5, 351, 1980.

AXFord, W. I., Solar Phys., 100, 575, 1985.

Belcher, J. W., Astrophys. J., 168, 509, 1971.

Belcher, J. W. and S. Olbert, Astrophys. J., 200, 369, 1975.

Breitschwerdt, D., J. F. MCKenzie, and H. J. VölK, Astron \& Astrophys., 245, 79, 1991.

Brueckner, G. E. and J. D. F. Bartoe, Astrophys. J., 272, 329, 1983. 
Brueckner, G. E., Adv. Space Res., 10, 161, 1990.

Courant, R. and K. O. Friedrichs, Supersonic Flow and Shock Waves, Interscience, New York, 1976.

TU, C.-Y., Solar Phys., 109, 149, 1987.

Davila, J. M., Astrophys. J., 317, 514, 1987.

Dere, K. P., J.-D. F. Bartel, and G. Brueckner, Astrophys. J., 345, L95, 1989.

Dere, K. P., Adv. Space Res., 10, 169, 1990.

Dougherty, M. K. and J. F. MCKenzie, J. Geophys. Res., 1990 (in press).

EsSer, R., E. Leer, S. R. HABbal, and G. L. Withbroe, J. Geophys. Res., 91, 2950, 1986.

ESSER, R., T. E. Holzer, and E. LeER, J. Geophys. Res., 92, 13377, 1987.

Gabriel, A. H., Phil Trans. R. Soc., A281, 339, 1976.

Habbal, S. R., J. F. Dowdy, Jr., and G. L. Withbroe, Astrophys. J., 352, 333, 1990.

Hollweg, J. V., Astrophys. J., 277, 392, 1981.

Hollweg, J. V., J. Geophys. Res., 91, 4111, 1986.

Hollweg, J. V. and W. Johnson, J. Geophys. Res., 93, 9547, 1988.

Holzer, T. E. and W. I. AXford, Ann. Rev. Astron Astrophys., 8, 30, 1970.

Isenberg, P. A. and J. W. Hollweg, J. Geophys. Res., 88, 3923, 1983.

Kojima, M., J. W. Amstrong, W. A. Coles, and B. J. Rickett, Solar Wind Six, NCAR/TN-306, 1, 53, 1987.

Kopp, R. A., Solar Wind Four, (MPAE-W-100-81-31), 42, 1981.

Kuperus, M. A., J. A. Ionson, and D. S. Spicer, Ann. Rev. Astron Astrophys., 19, 7, 1981.

LANDAU, L. D. and E. M. Lifshitz, Fluid Mechanics, Pergamon Press Oxford, 1966.

Leer, E. and T. E. Holzer, J. Geophys. Res., 85, 4681, 1980.

Leer, E., T. E. Holzer, and T. FlA, Space Sci. Rev., 33, 161, 1982.

Leer, E. and T. E. Holzer, Astrophys. J., 358, 680, 1990.

Mandrini, C. H., M. E. Machado, A. M. Hernandez, and M. G. Rovira, Adv. Space Res., 10, 115, 1990.

Mariska, J. T., Astrophys. J. 319, 465, 1989, Astrophys. J., 345, L95, 1989.

McKenZIE, J. F., W.-H. Ip, and W. I. Axford, Astrophys. Space Sci., 64 183, 1979.

McKenzie, J. F. and E. Marsch, Astrophys. Space Sci., 81, 295, 1982.

McKenzie, J. F., D. Breitschwerdt, and H. J. VÖLK, 20th ICRC 2 119, 1987.

NAIDU, K. and J. F. MCKenZIE, 1991 (in preparation).

Neugebauer, M., Solar Wind Five, NASA Publication 2280, 135, 1983.

PARKer, E. N., Interplanetary Dynamical Processes, Interscience, New York, 1963.

PARKer, E. N., Astrophys. J., 318, 876, 1987.

Parker, E. N., Adv. Space Res., 10 17, 1990.

Roberts, D. A., J. Geophys. Res., 94, 6899, 905, 1989.

TU, C.-H., Solar Phys., 109, 149, 1987.

Withrroe, G. L., Astrophys. J., 325, 442, 1988. 